\title{
In vivo Chemical Imaging and Quantitation of Microalgae Using Synchrotron- Based Infrared Microspectroscopic Imaging
}

\author{
S. Ratti*, C. Olivieri*, M. Nasse****, E. Duranty***, R. Burke***, Mario Giordano****, Frank \\ Vogt*** and C. J. Hirschmugl* \\ *Department of Physics, University of Wisconsin-Milwaukee, Milwaukee, WI \\ **Synchrotron Radiation Center, University of Wisconsin-Madison, Stoughton, WI \\ ***Department of Chemistry, University of Tennessee-Knoxville, Knoxville, TN \\ **** Faculty of Sciences, Polytechnical University of Marche, Ancona, ITALY
}

A combination of novel infrared imaging instrumentation [1] and advanced multivariate statistics [2] produces quantitative information for spatially resolved images of microalgae. In vivo chemical imaging of a common diatom Thallasosira weissfloggi demonstrates the capabilities of an infrared synchrotron beamline IRENI (Infrared Environmental Imaging) at the Synchrotron Radiation Center (Stoughton, WI) when used in combination with a newly designed flow chamber to maintain living cells in a controlled environs. IRENI has been designed to extract a swath of 12 beams of radiation from the synchrotron, optically recombine them into a single bundle of collimated beams, refocusing them with a Bruker Hyperion Microscope onto a sample area of 40x60 microns ${ }^{2}$, illuminating a 128 x128 pixel Focal Plane Array detector. The flow chamber [3] incorporates several important features that make diffraction-limited, spatially resolved, imaging of living cells feasible as demonstrated by studies on algae exposed to varying growing conditions. Chemomagic is a novel, user-friendly chemometrics program based on Principal Regression Analysis[4-6] and data pre-processing methods for quantitative analyses of target compounds in microalgae cells. For calibration purposes, an extensive spectroscopic calibration database of concentration series of 30 compounds with a total of $1400+$ well characterized samples has been implemented; it facilitates determining the concentration distributions of carbohydrates, proteins and lipids in individual living cells.

In Figure 1, we show two examples of predicting concentrations of standard test samples from the extensive database for Principal Component Regression calibration. Half of the database was used as the calibration model and the other half as the test spectra whose concentrations were then predicted. In each panel, the black dashed lines are lines corresponding to predicted concentration $=$ true concentration, and the black dots represent the predicted concentrations versus true concentrations for test samples of six individual analytes. The concentrations and error bars for the remaining 29 analytes not present in these test samples are represented enmass by the red dots, error bars and dashed lines; all predicted concentrations are close to zero as expected. In Figure 2, the calibration spectra were used in a Principal Component Analysis to demonstrate the comprehensiveness of the database for analyses of biological materials in general and microalgae in particular, where a reconstruction algorithm was applied to spectra obtained from E. Coli B and Scenedesmus subspicatus. The reconstructed spectra are compared to the original measured spectra; residual spectra are calculated from the difference between the measured spectrum and the reconstructed spectrum, establishing a measure of similarity between the two spectra (red, Figure 2). In Figure 3, a series of in vivo chemical images for single cells of Thallasosira weissfloggi grown under varying $\mathrm{N}$ conditions are shown. The images of the protein distribution (red/blue is high/low concentration) in the algae represent several points during the experimental time period, and the spectra show average results (central panel) over the algae from the series of experiments over 2 hours, and individual spectra (top and bottom panels) within the two selected images. 
References

[1] M.J. Nasse, et al., Nuclear Instruments and Methods in Physics Research A 582 (2007) 107.

[2] N. Draper et al., Applied Regression Analysis, 3rd ed., John Wiley \& Sons, New York, 1998.

[3] M.J. Nasse, et al., Applied Spectroscopy 63 (2009) 1180.

[4] Martens H, Næs T. , Multivariate Calibration, 2nd ed., John Wiley \& Sons: New York, 1991.

[5] Malinowski E. Factor Analysis in Chemistry, 3rd ed., John Wiley and Sons: New York, 2002.

[6] Gilbert MK, et al., Journal of Chemical Education. 85 (2008) 135.

[7] This research was carried out at IRENI (developed with funding from NSF-DMR-0619759) at the Synchrotron Radiation Center, which is funded by NSF-DMR-0537588. The research was supported by NSF-CHE-0832298 and the Research Grants Initiative program at UWMilwaukee.

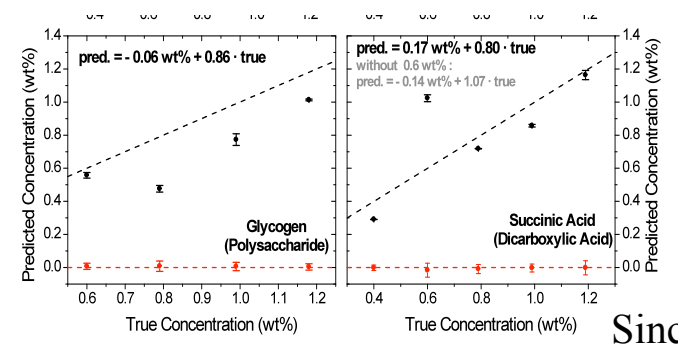

Figure 1: Predicted versus true concentrations of two analytes representing relevant compound groups typically found in biomaterials. Here, 30 analytes contained in a bio-database were included in the calibration. Approximately half of the database was utilized for this calibration; the remaining part was utilized as unknowns. only the analyte concentration was expected to be unequal to zero; the other analytes' predictions were expected to be zero. Thus, each panel depicts predicted versus true concentration for the unknown, individual analyte spectra (black dots and black dashed line); the remaining 29 analytes were expected to result in a zero concentration (red dots). For most analytes, the concentration predictions were rather precise; the not-present analytes were reliably detected as such.

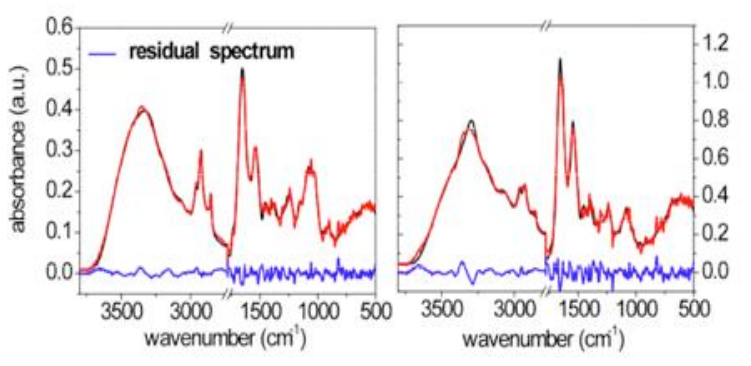

Figure 2: Demonstrating the comprehensiveness of the database - (red) reconstruction of (black) an algae spectrum, Scenedesmus subspicatus, (left panel) and an $E$. coli $B$ spectrum (right panel) by single-analyte spectra currently contained in the spectral database.

Figure 3. Time dependent series of infrared images and spectra for an algal cell maintained in
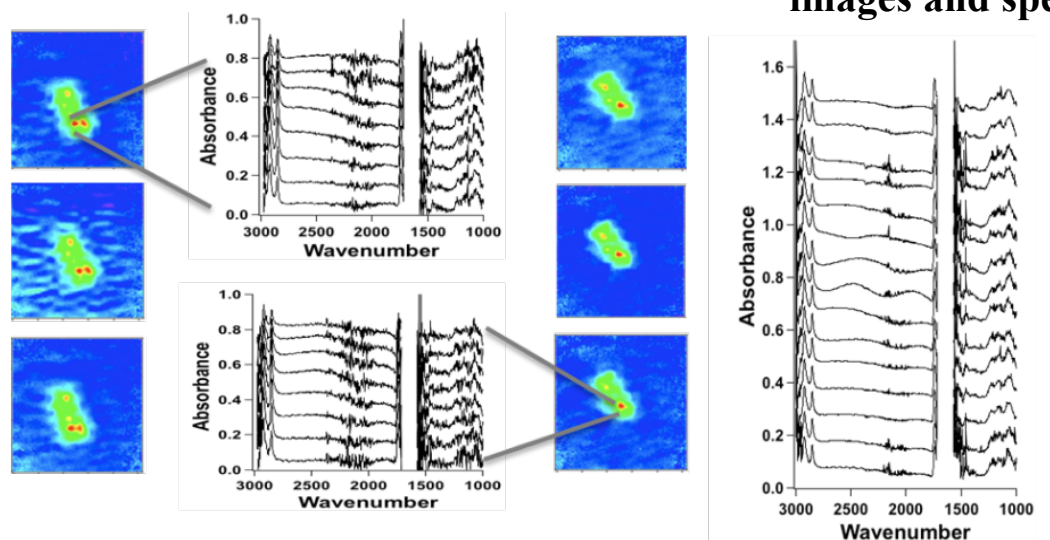
$\mathbf{N}$ rich and $\mathbf{N}$ replete media. Average spectra (shown in right panel) show subtle changes in the overall algal spectra as a function of $\mathrm{N}$ exposure. Individual pixel data show variations in local chemistry. The images show no change in internal structure during the Nitrogen rich experiments (left column), then a change in the structure between the N Rich and $\mathrm{N}$ replete exposure, followed by an apparently static distribution during the remainder of the $\mathrm{N}$ replete (right column) exposure. 